\title{
Essential genes*
}

\author{
Kenneth Kemphues ${ }^{\S}$, Department of Molecular Biology and Genetics, \\ Cornell University, Ithaca, NY, 14853, USA
}

\section{Table of Contents}

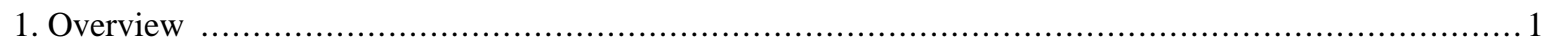

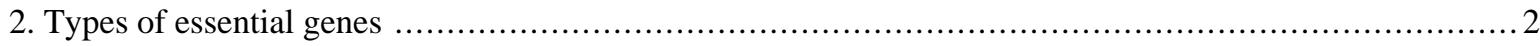

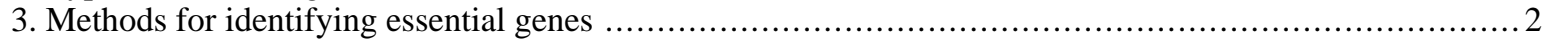

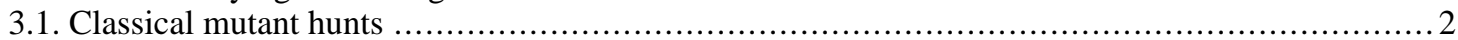

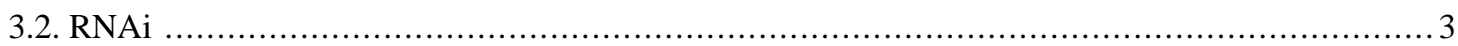

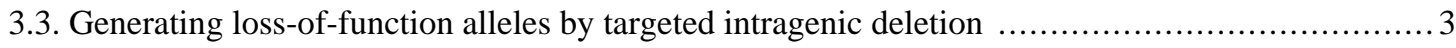

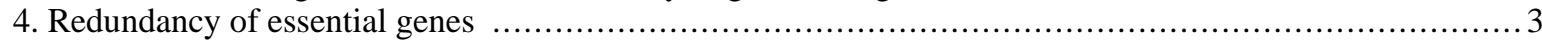

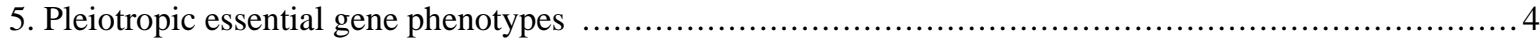

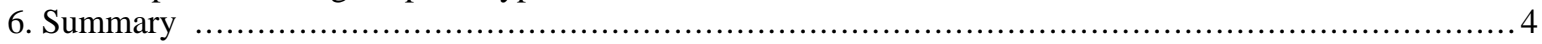

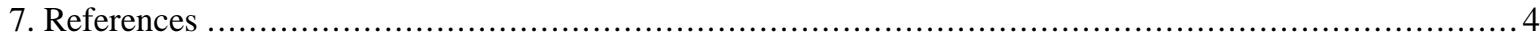

\begin{abstract}
Understanding the biology of $C$. elegans relies on identification and analysis of essential genes, genes required for growth to a fertile adult. Approaches for identifying essential genes include several types of classical forward genetic screens, genome-wide RNA interference screens and systematic targeted gene knockout. Based on most estimates made from screening results thus far, from 15-30\% of C. elegans genes appear to be essential. Genetic redundancy masks some essential functions and pleiotropy of many essential genes poses a challenge for a full understanding of their functions. Temperature sensitive mutations are valuable tools for studies of essential genes, but our ability to analyze essential genes would benefit from development of new tools for conditional inactivation or activation of specific genes.
\end{abstract}

\section{Overview}

Understanding the biology of $C$. elegans relies on identification and analysis of essential genes. An essential gene is defined here as a gene necessary for growth to a fertile adult. Some essential genes were identified fortuitously, but most essential genes have been identified through mutant screens designed specifically to isolate lethal and sterile mutations. Starting with Sydney Brenner's pioneering genetic analysis, many C. elegans geneticists have sought to understand the nature of the genome by estimating the fraction of the genome devoted to essential functions. Based on the frequency of X chromosome lethal mutations, Brenner estimated that the C. elegans genome had about 2000 essential genes (Brenner, 1974). Inclusion of sterile mutations raised this estimate to about 3000,

\footnotetext{
*Edited by Jonathan Hodgkin and Philip Anderson. Last revised March 4, 2005. Published December 24, 2005. This chapter should be cited as: Kemphues K. Essential Genes (December 24, 2005), WormBook, ed. The C. elegans Research Community, WormBook, doi/10.1895/wormbook.1.57.1, http://www.wormbook.org.

Copyright: (C) 2005 Kenneth Kemphues, This is an open-access article distributed under the terms of the Creative Commons Attribution License, which permits unrestricted use, distribution, and reproduction in any medium, provided the original author and source are credited.

${ }^{\S}$ To whom correspondence should be addressed. E-mail: kjk1@ cornell.edu
} 
and a number of screens for lethal and sterile mutations in specific genomic regions in the 1970s and 80s yielded gene frequencies raising the upper limit of this estimate to as high as $30 \%$ of the genome or about 5700 . (See Herman, 1978 and Johnsen and Baillie, 1997) for references and discussion. Also see (Feichtinger, 1995, for alternative statistical analyses arriving at an estimate of about 8500.) With the sequencing of the genome and the development of methods to target specific genes, it is now within our power to obtain loss-of-function mutations in every gene in the worm, and efforts are under way to do just that. However, even with this new ability to systematically knock out genes, many essential functions can remain cryptic due to genetic redundancy or to pleiotropy.

\section{Types of essential genes}

There are three types of mutations that identify essential functions: zygotic lethal mutations (lethals), maternal-effect lethal mutations (maternal-effect lethals) and sterile mutations (steriles). Zygotic lethals prevent the development to adult of individuals homozygous for the mutation. Zygotic lethals are broadly categorized, based on the time of developmental arrest, as embryonic or larval lethals. Maternal-effect lethals are a special class of sterilizing mutations that prevent the development of the progeny of hermaphrodites homozygous for the mutation. Such mutations define genes whose expression in the mother is required for embryonic development. Sterile mutations prevent the production of fertilized eggs by individuals homozygous for the mutation. Sterility could arise due to defects in germline development, somatic gonad development, oogenesis, spermatogenesis, ovulation or fertilization.

\section{Methods for identifying essential genes}

Classically, most lethal and sterile mutations have been identified by random mutagenesis followed by either of two types of screens: genome-wide screens for conditional lethals, such as temperature-sensitive mutations, and screens for non-conditional lethals and steriles in particular genomic regions for which balancers are available. More recently this approach has been augmented and, to a certain extent, replaced by approaches that target individual genes for knockdown or knockout. Two different large-scale screening methods are being used: systematic RNA interference (RNAi) and PCR-based screens for intragenic deletions after mutagenesis.

\subsection{Classical mutant hunts}

Genome wide screens for temperature-sensitive embryonic lethal mutations carried out in the late 1970s and early 80s provided the first stocked lethal mutants for studies of development. Genes identified in these early screens are named either $\mathrm{emb}$ (arrest in embryogenesis) or zyg (zygote defective) depending on the lab where the work was done, but operationally the screens were identical. Their analysis provided a foundation for understanding the complexity of lethal phenotypes as well as insight into the relative contributions of maternal vs. zygotic gene expression to the developmental process (see Wood, 1988 for references and discussion). Conclusions from the work were that many genes are required at multiple times in development and maternal gene expression plays a major role in embryogenesis. Temperature-sensitive lethal mutations are extremely valuable because they can be used to analyze essential genes with roles in multiple processes and are discussed more below.

Non-conditional lethal and sterile mutations must be maintained in the heterozygous condition, requiring constant selection to avoid loss of the mutation. This problem can be partially overcome by using closely linked visible markers to "balance" the lethal mutation. Because of this, early screens for lethal and sterile mutations were limited to small regions of the genome with convenient visible markers (e.g., Rogalski et al., 1982; Rose and Baillie, 1980). However, the development of free duplications and crossover-suppressing chromosomal rearrangements (balancer chromosomes) allowed easy maintenance of lethals and steriles, thus making it feasible to carry out large-scale screens for non-conditional lethal mutations (see Edgley et al., 1995 for a detailed discussion of balancer chromosomes). Such screens are restricted to regions of the genome for which balancers are available. However, because such balancers cover much of the genome it has been possible to collect mutations representing more than 860 essential loci by this method (for example Clark et al., 1988; Gonczy et al., 1999; Herman, 1978; Howell et al., 1987; Howell and Rose, 1990; Johnsen and Baillie, 1988; Johnsen and Baillie, 1991; Kemphues et al., 1988; McKim et al., 1988; McKim et al., 1992; Meneely and Herman, 1979; Rogalski et al., 1982; Rose and Baillie, 1980; Rosenbluth et al., 1983; Sigurdson et al., 1984; Stewart et al., 1998; Zetka and Rose, 1992). Genes identified in these screens are named let (lethal), ooc (oocyte defective), mel (maternal-effect lethal), and spe (spermatogenesis defective) or fer (fertilization defective - identical to spe mutants in phenotype). For mel and spe mutants it has been possible to develop genome-wide screens for non-conditional mutations in the absence of balancer 
chromosomes. In these screens, F1 progeny of mutagenized hermaphrodites are picked singly onto plates and the phenotypes are scored in the F2. In the case of spermatogenesis defective mutations, the homozygotes are identified as hermaphrodites that produce only unfertilized eggs and whose embryo production can be rescued by mating to wild-type males (for example Argon and Ward, 1980; L'Hernault et al., 1988; Ward and Miwa, 1978). In the case of mel mutations, the homozygous mutant animals are identified in the genetic background of an egg-laying defective mutation (Kemphues et al., 1988; Priess et al., 1987). Because egg-laying defects allow larvae to hatch inside the mother, killing her at a young age, mutations that block production of larval progeny allow survival of the F2 animals into old age. Maternal-effect lethal mutations can be distinguished from sterile mutations by the accumulation of fertilized but unhatched eggs in the uterus. Unfertilized eggs do not form light-refracting eggshells.

\subsection{RNAi}

Identification and analysis of essential genes has been greatly facilitated by RNA interference (RNAi; see Reverse genetics).RNAi leads to gene-specific mRNA degradation and is triggered by double stranded RNAi specific for the target gene. The double stranded RNA can be delivered by microinjection, by uptake of dsRNA by worms in solution (soaking), or by feeding worms bacteria engineered to synthesize the RNA of interest. Whole genome screens have been carried out by RNAi feeding in a wild-type genetic background (Fraser et al., 2000; Kamath et al., 2003) and in the rrf-3 sensitized genetic background (Simmer et al., 2003). Because RNAi is susceptible to false negatives and often gives incompletely penetrant phenotypes (see below), it can only provide a minimum estimate for essential genes in the genome. Although the screens in the rrf-3 genetic background were more sensitive for viable RNAi phenotypes, they identified slightly fewer essential genes than found in the wild-type background. Based on RNAi results in the wild-type background, the minimum number of essential genes is about 1750. (1170 genes with lethal and sterile RNAi phenotypes adjusted for a false negative rate of $22 \%$ and $86 \%$ genome coverage; Kamath et al., 2003) Screens have also been carried out using RNAi administered by injection or soaking worms in dsRNA solutions (Gonczy et al., 2000; Maeda et al., 2001; Piano et al., 2000; Piano et al., 2002). Comparison of these results to each other and to results from classical genetic screens has revealed some limitations of RNAi for discovering gene function: some genes appear to be insensitive to RNAi and outcomes can vary. Some essential genes will give no RNAi phenotypes, give very weak phenotypes or give phenotypes other than lethality or sterility. Furthermore, the efficiency of RNAi differs somewhat with different techniques for introducing the dsRNA triggers (Piano et al., 2002) and even from trial to trial with the same technique (Simmer et al., 2003). Another limitation, not restricted to RNAi, is genetic redundancy (discussed below and see Gene duplications and genetic redundancy in C. elegans).

\subsection{Generating loss-of-function alleles by targeted intragenic deletion}

Two approaches have been used to generate targeted loss-of-function mutations by intragenic deletion. Common to both is the PCR-based screening of populations of randomly mutagenized worms to identify rare deletions in targeted genes. The worms carrying the deletions are enriched and eventually isolated by sibling selection schemes. Initially, transposon insertions in targeted genes were isolated in the expectation that the insertions would be mutagenic (Rushforth et al., 1993), but because of a high frequency of silent insertions (Rushforth and Anderson, 1996; Rushforth et al., 1993) a two-step process of first isolating insertion-bearing worms and then screening populations of these worms for imprecise excision events was developed (Zwaal et al., 1993). Chemical mutagenesis, which proved to be more efficient, has now replaced the use of transposon excision for generating deletions (Jansen et al., 1997). The procedure is labor-intensive, and not efficient for a single target gene. Therefore, two groups have organized large scale efforts and are now generating intragenic deletions based on requests from members of the research community: the C. elegans Gene Knockout Consortium and the National Bioresource Project for the Experimental Animal C. elegans Based on results to date, about 1800 genes have been knocked out with 20-24\% being essential (Shohei Mitani, Don Moerman and Bob Barstead, personal communications). Projecting the higher frequency to the whole genome (19,727 CDS; Wormbase, release WS136, 12/3/05) yields an estimate of 4645 essential genes. However, this is likely to be inaccurate because gene knockouts are not being done randomly, but rather are biased toward genes with known functions in other organisms, or towards genes with known mutant or RNAi phenotypes.

\section{Redundancy of essential genes}

Many genes are members of multigene families and members of these families can often have redundant functions. In rare cases where nucleotide sequence is highly conserved, RNAi with a single trigger molecule can target multiple family members (e.g., Colombo et al., 2003). If the number of family members is small, it is possible to reveal redundancies by double or triple knockout or knockdown (e.g., Schubert et al., 2000; Gotta and Ahringer, 
2001). Redundant pathways, in which different processes can accomplish the same essential function, are more cryptic and are not likely to be detected except by fortuitous double mutant combinations or screens for synthetic lethal mutations (enhancers). See Gene duplications and genetic redundancy in C. elegans for further consideration of redundancy and Genetic enhancers for further discussion of enhancers.

\section{Pleiotropic essential gene phenotypes}

Many essential genes function repeatedly during the life of the worm and can have different essential roles in different tissues. (See Genetic mosaics) However, null mutant phenotypes often reveal only one essential role, leading to incomplete understanding of the gene's role. A clear example is the Notch family member $g l p-1$. Null mutations in $g l p-1$ are sterile mutations. Analysis of the null alleles revealed that $g l p-l$ was required for germline proliferation (Austin and Kimble, 1987). Expression of $g l p-1$ during oogenesis is also required for cell fate specification in the early embryo. However, this function is masked in animals homozygous for the null allele by the failure of these animals to produce embryos. The embryonic role of $g l p-1$ was revealed by the recovery of $e 2072$, a special non-conditional allele that did not affect germline proliferation, and three temperature-sensitive mutations (Priess et al., 1987). Analysis of these mutations revealed that signaling through the GLP-1 protein plays major roles in cell fate specification at multiple times in the early embryo (Hutter and Schnabel, 1994; Mello et al., 1994; Moskowitz et al., 1994; Priess et al., 1987). Clearly, null alleles generated by targeted deletions will be inadequate for full understanding of essential gene functions.

The glp- 1 example illustrates not only the problem of pleiotropy but also one solution - the recovery and analysis of temperature-sensitive mutations. Temperature-sensitive mutations, as discussed above, are being used effectively for analysis of many essential $C$. elegans genes. Judicious temperature shifts can reveal multiple functions for a given gene. For example, shifting from permissive to restrictive temperature for brief periods early in embryogenesis revealed distinct roles for $g l p-1$ at the four-cell and the 12-cell stage (Mello et al., 1994). Therefore the continued screening for temperature-sensitive lethal mutations (e.g., Encalada et al., 2000; Golden et al., 2000; O'Connell et al., 1998) will provide reagents for specific analyses that often benefit the community at large. The relative rarity of temperature-sensitive mutations and the extreme difficulty of targeting such mutations to specific genes restricts their utility.

Another solution to the pleiotropy problem, conditional gene expression, used effectively in other genetic models like fruit flies and mice, has not yet been developed for $C$. elegans. In this approach, transgenes that have conditional promoters driving the gene of interest or that have strategically-placed recombination sequences are transformed into null mutant backgrounds. These constructs can be designed for gene activation or inactivation in specific tissues, at specific developmental times or in response to drug applications. Systems for conditional gene expression include the GAL4-UAS system applied effectively in fruit flies (Duffy, 2002) and the Cre/lox and tetracycline inducible systems being used in mouse (Gossen and Bujard, 2002). Adaptation of these tools for $C$. elegans would greatly facilitate analysis of essential genes.

\section{Summary}

The number of essential genes in C. elegans as estimated by gene knockout, RNAi and classical genetic screens appears to be less than $30 \%$ of the genome. The combination of RNAi and targeted gene knockouts ensures the identification of most essential C. elegans genes in the foreseeable future. Genetic redundancy could mask a significant number of essential functions, but construction of double and triple mutants or polygenic RNAi has the potential to identify many of these cases. Studies of null mutations can identify many roles of essential genes, but some roles of essential genes can be masked by early developmental arrest. Although temperature-sensitive mutations can overcome this problem, the difficulty of targeting temperature-sensitive mutations to specific genes makes it desirable to develop additional tools for conditional inactivation or activation of essential genes.

\section{References}

Argon, Y., and Ward, S. (1980). Caenorhabditis elegans fertilization-defective mutants with abnormal sperm. Genetics 96, 413-433. Abstract

Austin, J., and Kimble, J. (1987). glp-1 is required in the germ line for regulation of the decision between mitosis and meiosis in C. elegans. Cell 51, 589-599. Abstract 
Brenner, S. (1974). The genetics of Caenorhabditis elegans. Genetics 77, 71-94. Abstract

Clark, D.V., Rogalski, T.M., Donati, L.M., and Baillie, D.L. (1988). The unc-22(IV) region of Caenorhabditis elegans: genetic analysis of lethal mutations. Genetics 119, 345-353. Abstract

Colombo, K., Grill, S.W., Kimple, R.J., Willard, F.S., Siderovski, D.P., and Gonczy, P. (2003). Translation of polarity cues into asymmetric spindle positioning in Caenorhabditis elegans embryos. Science 300, 1957-1961. Abstract Article

Duffy, J.B. (2002). GAL4 system in Drosophila: a fly geneticist's Swiss army knife. Genesis 34, 1-15. Abstract Article

Edgley, M.L., Baillie, D.L., Riddle, D.L., and Rose, A.M. (1995). Genetic balancers. Methods Cell Biol. 48, 147-184. Abstract

Encalada, S.E., Martin, P.R., Phillips, J.B., Lyczak, R., Hamill, D.R., Swan, K.A., and Bowerman, B. (2000). DNA replication defects delay cell division and disrupt cell polarity in early Caenorhabditis elegans embryos. Dev. Biol. 228, 225-238. Abstract Article

Feichtinger, R. (1995) Quantitative analysis of maternal gene functions of Caenorhabditis elegans. University of Vienna, Austria, Vienna.

Fraser, A.G., Kamath, R.S., Zipperlen, P., Martinez-Campos, M., Sohrmann, M., and Ahringer, J. (2000). Functional genomic analysis of $C$. elegans chromosome I by systematic RNA interference. Nature 408, 325-330. Abstract Article

Golden, A., Sadler, P.L., Wallenfang, M.R., Schumacher, J.M., Hamill, D.R., Bates, G., Bowerman, B., Seydoux, G., and Shakes, D.C. (2000). Metaphase to anaphase (mat) transition-defective mutants in Caenorhabditis elegans. J. Cell Biol. 151, 1469-1482. Abstract

Gonczy, P., Echeverri, G., Oegema, K., Coulson, A., Jones, S.J., Copley, R.R., Duperon, J., Oegema, J., Brehm, M., Cassin, E., et al. (2000). Functional genomic analysis of cell division in C. elegans using RNAi of genes on chromosome III. Nature 408, 331-336. Abstract Article

Gonczy, P., Schnabel, H., Kaletta, T., Amores, A.D., Hyman, T., and Schnabel, R. (1999). Dissection of cell division processes in the one cell stage Caenorhabditis elegans embryo by mutational analysis. J. Cell Biol. 144, 927-946. Abstract

Gossen, M., and Bujard, H. (2002). Studying gene function in eukaryotes by conditional gene inactivation. Annu. Rev. Genet. 36, 153-173. Abstract Article

Gotta, M., and Ahringer, J. (2001). Distinct roles for G $\alpha$ and G $\beta \gamma$ in regulating spindle position and orientation in Caenorhabditis elegans embryos. Nat. Cell Biol. 3, 297-300. Abstract Article

Herman, R.K. (1978). Crossover suppressors and balanced recessive lethals in Caenorhabditis elegans. Genetics 88, 49-65. Abstract

Howell, A.M., Gilmour, S.G., Mancebo, R.A., and Rose, A.M. (1987). Genetic analysis of a large autosomal region in Caenorhabditis elegans by the use of a free duplication. Genet. Res. 49, 207-213.

Howell, A.M., and Rose, A.M. (1990). Essential genes in the hDf6 region of chromosome I in Caenorhabditis elegans. Genetics 126, 583-592. Abstract

Hutter, H., and Schnabel, R. (1994). glp-1 and inductions establishing embryonic axes in C. elegans. Development 120, 2051-2064. Abstract

Jansen, G., Hazendonk, E., Thijssen, K.L., and Plasterk, R.H. (1997). Reverse genetics by chemical mutagenesis in Caenorhabditis elegans. Nature Genetics 17, 119-121. 
Johnsen, R.C., and Baillie, D.L. (1988). Formaldehyde mutagenesis of the eTl balanced region in Caenorhabditis elegans: dose-response curve and the analysis of mutational events. Mutat. Res. 201, 137-147. Abstract

Johnsen, R.C., and Baillie, D.L. (1991). Genetic analysis of a major segment [LGV(left)] of the genome of Caenorhabditis elegans. Genetics 129, 735-752. Abstract

Johnsen, R.C., and Baillie, D.L. (1997). Mutation. In C. elegans II, D.L. Riddle, T. Blumenthal, B.J. Meyer, and J.R. Priess, eds., Cold Spring Harbor, Cold Spring Harbor Laboratory Press, pp. 79-95.

Kamath, R.S., Fraser, A.G., Dong, Y., Poulin, G., Durbin, R., Gotta, M., Kanapin, A., Le Bot, N., Moreno, S., Sohrmann, M., et al. (2003). Systematic functional analysis of the Caenorhabditis elegans genome using RNAi. Nature 421, 231-237. Abstract Article

Kemphues, K.J., Kusch, M., and Wolf, N. (1988). Maternal-effect lethal mutations on linkage group II of $C$. elegans. Genetics 120, 977-986. Abstract

Kemphues, K.J., Priess, J.R., Morton, D.G., and Cheng, N.S. (1988). Identification of genes required for cytoplasmic localization in early C. elegans embryos. Cell 52, 311-320. Abstract

L'Hernault, S.W., Shakes, D.C., and Ward, S. (1988). Developmental genetics of chromosome I spermatogenesis-defective mutants in the nematode Caenorhabditis elegans. Genetics 120, 435-452. Abstract

Maeda, I., Kohara, Y., Yamamoto, M., and Sugimoto, A. (2001). Large-scale analysis of gene function in Caenorhabditis elegans by high-throughput RNAi. Curr. Biol. 11, 171-176. Abstract

McKim, K.S., Heschl, M.F., Rosenbluth, R.E., and Baillie, D.L. (1988). Genetic organization of the unc-60 region in Caenorhabditis elegans. Genetics 118, 49-59. Abstract

McKim, K.S., Starr, T., and Rose, A.M. (1992). Genetic and molecular analysis of the dpy-14 region in Caenorhabditis elegans. Mol. Gen. Genet. 233, 241-251. Abstract

Mello, C.C., Draper, B.W., and Priess, J.R. (1994). The maternal genes apx-1 and glp-1 and establishment of dorsal-ventral polarity in the early C. elegans embryo. Cell 77, 95-106. Abstract

Meneely, P.M., and Herman, R.K. (1979). Lethals, steriles and deficiencies in a region of the X chromosome of Caenorhabditis elegans. Genetics 92, 99-115. Abstract

Moskowitz, I.P., Gendreau, S.B., and Rothman, J.H. (1994). Combinatorial specification of blastomere identity by glp-1-dependent cellular interactions in the nematode Caenorhabditis elegans. Development 120, 3325-3338. Abstract

O'Connell, K.F., Leys, C.M., and White, J.G. (1998). A genetic screen for temperature-sensitive cell-division mutants of Caenorhabditis elegans. Genetics 149, 1303-1321. Abstract

Piano, F., Schetter, A.J., Mangone, M., Stein, L., and Kemphues, K.J. (2000). RNAi analysis of genes expressed in the ovary of Caenorhabditis elegans. Curr. Biol. 10, 1619-1622. Abstract

Piano, F., Schetter, A.J., Morton, D.G., Gunsalus, K.C., Reinke, V., Kim, S.K., and Kemphues, K.J. (2002). Gene clustering based on RNAi phenotypes of ovary-enriched genes in C. elegans. Curr. Biol. 12, 1959-1964. Abstract

Priess, J.R., Schnabel, H., and Schnabel, R. (1987). The glp-1 locus and cellular interactions in early C. elegans embryos. Cell 51,601-611. Abstract

Rogalski, T.M., Moerman, D.G., and Baillie, D.L. (1982). Essential genes and deficiencies in the unc-22 IV region of Caenorhabditis elegans. Genetics 102, 725-736. Abstract

Rose, A.M., and Baillie, D.L. (1980). Genetic organization of the region around UNC-15 (I), a gene affecting paramyosin in Caenorhabditis elegans. Genetics 96, 639-648. Abstract 
Rosenbluth, R.E., Cuddeford, C., and Baillie, D.L. (1983). Mutagenesis in Caenorhabditis elegans. I. A rapid eukaryotic mutagen test system using the reciprocal translocation eTI (III, V). Mutat. Res. 110, 39-48.

Rushforth, A.M., and Anderson, P. (1996). Splicing removes the Caenorhabditis elegans transposon Tc1 from most mutant pre-mRNAs. Mol. Cell Biol. 16, 422-429. Abstract

Rushforth, A.M., Saari, B., and Anderson, P. (1993). Site-selected insertion of the transposon Tc1 into a Caenorhabditis elegans myosin light chain gene. Mol. Cell Biol. 13, 902-910. Abstract

Schubert, C.M., Lin, R., de Vries, C.J., Plasterk, R.H., and Priess, J.R. (2000). MEX-5 and MEX-6 function to establish soma/germline asymmetry in early C. elegans embryos. Mol. Cell 5, 671-682. Abstract

Sigurdson, D.C., Spanier, G.J., and Herman, R.K. (1984). Caenorhabditis elegans deficiency mapping. Genetics 108, 331-345. Abstract

Simmer, F., Moorman, C., van der Linden, A.M., Kuijk, E., van den Berghe, P.V., Kamath, R.S., Fraser, A.G., Ahringer, J., and Plasterk, R.H. (2003). Genome-wide RNAi of C. elegans using the hypersensitive rrf-3 strain reveals novel gene functions. PLoS Biol. 1, E12. Abstract Article

Stewart, H.I., O'Neil, N.J., Janke, D.L., Franz, N.W., Chamberlin, H.M., Howell, A.M., Gilchrist, E.J., Ha, T.T., Kuervers, L.M., Vatcher, G.P., et al. (1998). Lethal mutations defining 112 complementation groups in a $4.5 \mathrm{Mb}$ sequenced region of Caenorhabditis elegans chromosome III. Mol. Gen. Genet. 260, 280-288. Abstract

Ward, S., and Miwa, J. (1978). Characterization of temperature-sensitive, fertilization-defective mutants of the nematode Caenorhabditis elegans. Genetics 88, 285-303. Abstract

Wood, W.B., ed. (1988). The Nematode Caenorhabditis elegans (Cold Spring Harbor, Cold Spring Harbor Laboratory Press).

Zetka, M.C., and Rose, A.M. (1992). The meiotic behavior of an inversion in Caenorhabditis elegans. Genetics 131, 321-332. Abstract

Zwaal, R.R., Broeks, A., Van, M.J., Groenen, J.T. M., and Plasterk, R.H. A. (1993). Target-selected gene inactivation in Caenorhabditis elegans by using a frozen transposon insertion mutant bank. Proc. Natl. Acad. Sci. USA 90, 7431-7435. Abstract

All WormBook content, except where otherwise noted, is licensed under a Creative Commons Attribution License 